\title{
Determining Factors and Critical Periods in the Formation of Eating Habits: Results from the Habeat Project
}

\author{
Sylvie Issanchou on behalf of the Habeat consortium \\ Centre des Sciences du Goût et de l'Alimentation, AgroSup Dijon, CNRS, INRA, University of Bourgogne \\ Franche-Comté, Dijon, France
}

\begin{abstract}
Keywords
Infant - Toddler · Pre-school children · Vegetable acceptance $\cdot$ Learning $\cdot$ Repeated exposure $\cdot$ Vegetable intake · Caloric compensation · Eating in the absence of hunger
\end{abstract}

\begin{abstract}
Background: Eating habits form early during childhood and are likely to track until the beginning of adulthood. Thus, understanding the formation of eating habits is important. Consequently, the population targeted in the European project Habeat comprised infants and young children up to 6 years of age. Summary: Habeat mainly focused on the qualitative dimension of eating habits with a particular attention on vegetables and to a lesser extent on fruit. Food intake of young children was also studied in 2 challenging situations where overeating may occur and the relation between children's behaviour and feeding parental practices was examined. Key Messages: Habeat found evidence that breastfeeding may facilitate the consumption of vegetables and fruit and a greater variety of healthy foods in later childhood. Introduction of a variety of vegetables at the beginning of the complementary feeding period increases later acceptance of novel foods. Repeated exposure is a powerful mechanism to increase children's intake of a novel vegetable in infants and young children. Offering energy-dense snacks before or after meals should be avoided. Moreover, food
\end{abstract}

\section{KARGER}

(c) 2017 S. Karger AG, Basel

E-Mail karger@karger.com

www.karger.com/anm should be offered to children in response to their feelings of hunger, and not used as reward for a good behaviour or for any other reason.

(c) 2017 S. Karger AG, Basel

\section{Introduction}

Recent surveys have shown that the diets of children in many European countries are not ideal. Different intervention studies based on nutritional information show that it is difficult to modify eating behaviours in children, teenagers and even more in adults. Studies so far have shown that preferences and eating habits form early during childhood and are likely to track until the beginning of adulthood [1-3]. Thus, understanding the early formation of eating habits is important. A large part of the European Habeat project (https://www.habeat.eu/) focused on the development of acceptance of vegetables and, to a lesser extent of fruit, because European children eat too few vegetables and fruit. Observational approaches, based on data from 4 European cohorts, and experimental approaches were used. The quantitative dimension of eating habits was also investigated. Food intake of young children, in 2 challenging situations where overeating may occur, was studied as well as the relation between children's eating behaviour and feeding parental practices. 


\section{Main Questions and Key Results}

Early Feeding Practices and Eating Habits in Childhood

We aimed to examine whether early feeding practices, such as breastfeeding duration and timing of complementary feeding, influence fruit and vegetable intake later in childhood. Four birth cohorts from France (EDEN), the United Kingdom (ALSPAC), Portugal (Generation XXI), and Greece (EuroPrevall) with comparable data on breastfeeding initiation and duration, age at which complementary feeding had started and early childhood diet were studied [4]. Parallel analyses with identical statistical models were performed in each country. Fruit and vegetable intake and healthy dietary variety were assessed thanks to food frequency questionnaires completed by parents for their child at different ages. Never or short breastfeeding duration ( $<3$ months) was quite consistently related to lower fruit and vegetable intake. Moreover, results from the United Kingdom indicated that this association could persist through childhood (Table 1). The results were similar when regressions were adjusted for maternal vegetable or fruit intake. Based on the analysis of data collected in EDEN, ALSPAC, and Generation XXI, it was also found that being breastfed for a short duration is associated with pre-school children eating a lower variety of healthy foods [5] (Table 1). The results on timing of complementary feeding were not consistent across the cohorts. Thus, this work has allowed us to conclude that never or short duration of breastfeeding but not timing of complementary feeding are associated with a less healthy diet in early childhood. The quite similar results obtained for different cohorts suggest that the benefits of breastfeeding on the development of healthy eating habits would not be country-specific.

\section{Effect of Exposure to Early Variety on Vegetable Acceptance}

Previous research has shown that early experience with different flavours leads to greater acceptance of unfamiliar foods [6]. It does not seem necessary to introduce many vegetables in the first weeks of complementary feeding and frequency of change appears more effective than the number of vegetables fed [7]. It was also observed in the Opaline cohort that the higher the number of foods introduced during the first 2 months of complementary feeding, the higher the new food acceptance beyond this 2-month period until the age of 15 months [8]. In Habeat, we investigated whether these findings have real-world application by testing a parent-led intervention to increase infants' vegetable acceptance [9]. Thus, mothers of 4- to 6-month-old infants in the United Kingdom, Greece, and Portugal were randomised to either an intervention group, who received guidance on introducing 5 vegetables (one per day) as first foods, repeated over 15 days, or a control group who received country-specific "usual care". Infant's consumption and liking (rated by mothers and researchers), of an unfamiliar vegetable were assessed one month post-intervention. Analyses showed that UK intervention infants consumed and liked significantly more the unfamiliar vegetable than control infants. In Portugal and Greece, there was no significant intervention effect. These different results may be explained by cultural differences in typical complementary feeding practices in the 3 countries. However, the results suggest that in countries, such as the United Kingdom, where vegetables are not common first foods, advice on introducing a variety of vegetables early in weaning may be beneficial for increasing vegetable acceptance. Fildes et al. [9] indicated that this "intervention was received positively by parents; they welcomed the simple, prescriptive, and unambiguous nature of the instructions". As a matter of fact, this intervention requires parents to simply select single vegetables as their child's first foods and to offer them on a daily rotation, persisting even when their child appears to dislike a food. The advice would be easy to disseminate to parents since they are quite anxious about complementary feeding stage, are seeking advice, and have contact with health professionals before starting introduction of solid foods.

\section{Learning to Eat Vegetables by Repeated Exposure and}

Role Modelling

Children learned to eat and like foods through many different ways. A first way of learning is to learn by experience. Thus, children may learn through repeated exposure, which consists of offering the same food several times without forcing the child. This repeated exposure will allow the child to recognize the food as safe if ingestion has no negative post-ingestive effects. Another type of learning by experience is the flavor-flavour learning. In this case, a neutral or disliked stimulus is temporarily paired with a positive stimulus, for example a sweet taste; this induces a positive shift in response to the initially neutral or disliked stimulus even when it is presented without the liked stimulus. A third type of learning by experience is the flavor-nutrient learning. In this case, a neutral or disliked stimulus is temporarily paired with calories that induce a positive post-ingestive effect; again, this induces a positive shift in response to the initially neutral or disliked stimulus even when it is presented without calories. Previous studies had shown that these different types of learning by experience may be effective to increase vegetable liking in children but none had compared the efficiency of these 3 strategies for increasing acceptance of a same vegetable in children within a small range of age. This was done in Habeat. All studies showed that repeated exposure to a vegetable is an effective strategy to increase the intake and liking of an unfamiliar vegetable among infants and young children [10-16] (Table 2). Concerning the generalisation of learning from one vegetable to another vegetable, there are conflicting results in both the literature and the Habeat results. However, Habeat results seem to indicate that generalisation of learning could occur when the sensory properties of a novel vegetable are very close to the sensory properties of the "learned" vegetable [10]. Despite the fact that repeated exposure might be effective for most children up to 3 years of age, about $15 \%$ of the children were considered "non-eaters" eating less than $10 \mathrm{~g}$ by the 5 th exposure; older pre-school and fussier children were more likely to be "non-eaters" [17]. Moreover, for 3 - to 5-year-old children, repeated exposure (twice a week) was not effective in increasing children's intake of a familiar vegetable with low initial consumption. Therefore, alternative strategies might be needed for older and/or fussier children. These techniques could be based on adding some desired seasoning, sauce or ingredient that might facilitate acceptance of a new vegetable in these children. It is also important to encourage parents to be persistent and patient and to recommend them to avoid pressurizing their child.

Previous research has shown that observational learning (role modelling) may play an important role in shaping children's food choice behaviour. Children learn about food by observing other people. Parents are the first role models for children, but peers, idols and teachers may also be models and children are likely to 


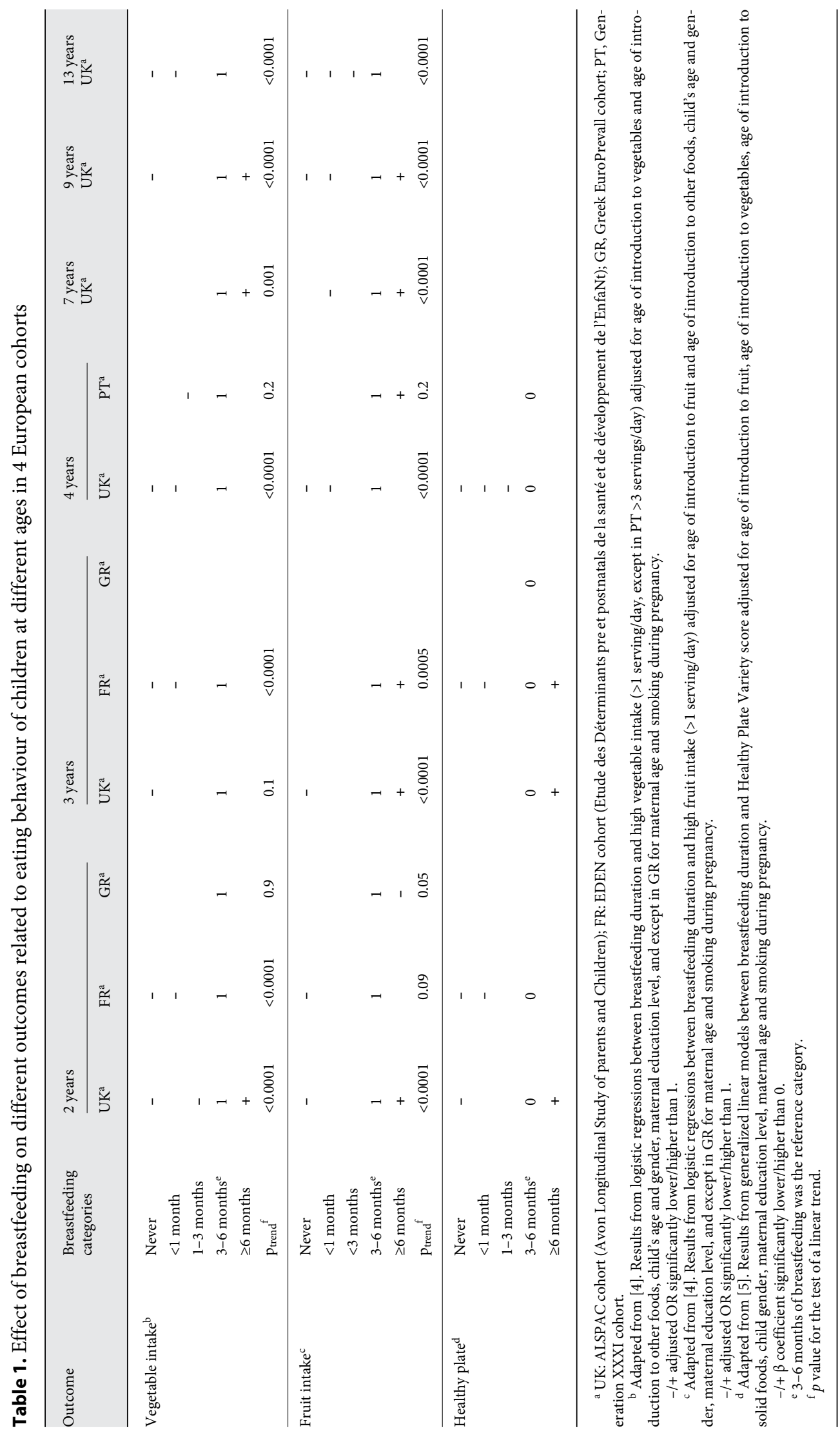




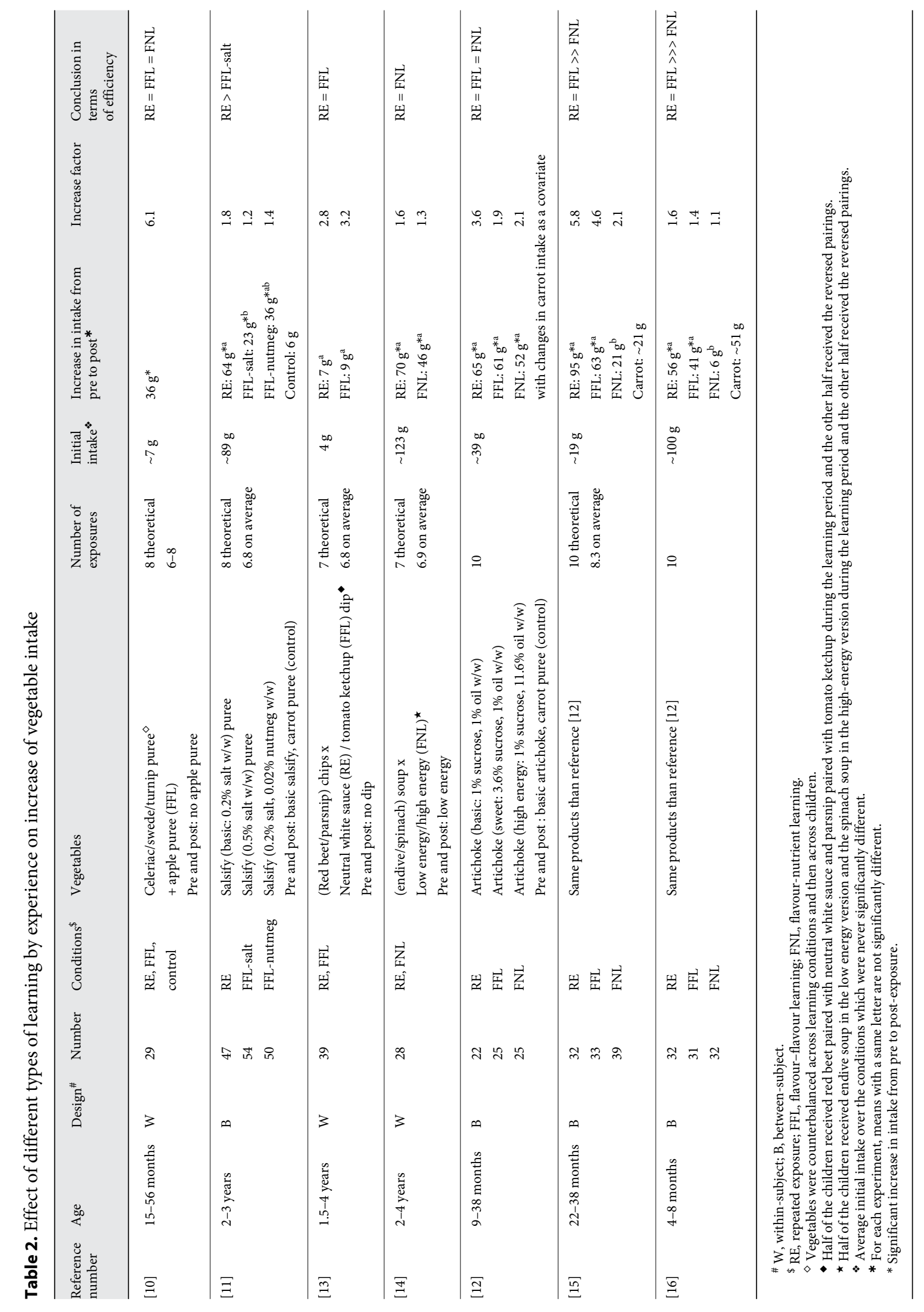


imitate their behaviour. This has been previously studied for new or unfamiliar food products, but less is known about the effect of role modelling on children's intake of a relatively familiar vegetable. One study showed an increased acceptance for previously less liked vegetables in children who were exposed to children who had a high preference for that target vegetable [18]. In Habeat, it was examined if role modelling by idols and teachers in school settings could increase the intake of familiar vegetables. Immediately after the intervention, intake did not increase. It is possible that the target vegetable was offered too frequently leading to a boredom effect, which counteracted the role modelling effect. However, a delayed effect of the classroom-based intervention was found since, 9 months later, children's average carrot intake had increased in the intervention groups, whereas it had remained stable for the control group [19].

\section{The Effect of Choice Offering on Children's Vegetable Intake}

According to the self-determination theory, choice should result in positive intrinsic motivation and consequently in higher performance and satisfaction. Transposed to the food domain, providing someone the opportunity to choose the food that she/ he wants to consume should elicit an increased motivation to eat, as well as greater food enjoyment. Only a few studies have investigated the influence of free choice on children's vegetable intake. These studies showed that children liked the practice of being allowed to choose, but the results regarding vegetable intake were not consistent $[20,21]$. Therefore, the effect of choice offering on children's vegetable intake was investigated in 3 countries. In Denmark and Greece, a within-subject design was used, with children aged 3-5 in a school/nursery setting. In the Netherlands, a between-subject design was used, with children aged 2-6 in the home-setting. Mixed results were found in Denmark and Greece, but in the Netherlands, the results suggest a small positive effect of choice offering on children's vegetable intake during dinner in an in-home situation [22]. New studies are necessary to determine the conditions that will result in the strengthening of the positive effect of the choice and so to give the parents advice, which will have more chance to lead to an increased vegetable intake.

\section{Involvement in Vegetable Preparation}

Previous studies suggest that cooking sessions may be a promising strategy to increase children's vegetable consumption. Involvement in food preparation should result in positive intrinsic motivation and consequently, might increase the perceived value and liking of the self-prepared food. However, participation in one interactive vegetable preparation session with an enthusiastic chefcook in a restaurant setting did not increase vegetable intake among children aged $4-6$. This result could be due to the fact that the dinner was served in an unfamiliar setting and/or to the fact that parents were requested not to focus on the vegetables in the parent-child conversations during the meal. A positive effect on intake may be expected if involvement in food preparation takes place at home and on the longer term.

\section{Control of Food Intake}

Between the ages of 3 and 6, children may become less responsive to internal cues of hunger and satiation, and more responsive to external food cues, which may induce overeating and contribute to weight gain. Thus, it is important to study children's behaviour in challenging situations where overeating may occur. For the first time, we examined 3- to 6-year-old children's intake in 2 situations: one where a food item is offered before a meal and another one where palatable food stuff were at their disposal after a meal [23]. The first situation corresponds to the paradigm of caloric compensation: we compared energy intake at 2 meals, one being preceded by the consumption of an energy dense snack. The second situation corresponds to the eating that happens in the absence of the hunger paradigm: we measured the energy intake during the snack in comparison to the energy intake during the meal. We found that when offered an energy-dense food before a lunch, children ate less. But, on average, they only reduced their lunch intake by half $(50 \%)$ the calories represented by the snack. We also observed that when palatable foods were offered after a lunch, the average intake was the equivalent of $25 \%$ of the energy intake of the lunch. Mothers' feeding practices were assessed using a questionnaire and we found that mothers who used "Food as a reward" were more likely to have children who ate in the absence of hunger. We developed an intervention targeted to children aged at least 4 with either poorer caloric compensation or higher intake in the absence of hunger than the median. This intervention aimed to help children focus on their internal cues of hunger and satiation to avoid overeating. However, we did not observe a significant impact of this intervention. Thus, teaching children to pay attention to their hunger and satiation cues is not easy and the best option is probably to avoid placing children in challenging situations where they may overeat.

\section{Conclusion}

The results of this project have been translated into recommendations addressed to health professionals, child care professionals and policy makers and published in a stakeholder brochure (available on https://www.habeat.eu/). An elaborate guide for parents of young children was developed on the basis of the project's results and of prior data [24]. This guide is available in 7 languages on the web site of the project.

\section{Funding}

The research leading to these results has received funding from the European Community's Seventh Framework Program (FP7/2007-2013) under the grant agreement $n^{\circ}$ FP7-245012-Habeat.

\section{Disclosure Statement}

The research group has received funds from Blédina (a brand of Danone) and from Nestlé Research Center for conducting research projects. 


\section{References}

1 Nicklaus S, Boggio V, Chabanet C, Issanchou $S$ : A prospective study of food preferences in childhood. Food Qual Pref 2004;15:805-818.

2 Nicklaus S, Boggio V, Chabanet C, Issanchou $\mathrm{S}$ : A prospective study of food variety seeking in childhood, adolescence and early adult life. Appetite 2005;44:289-297.

3 Skinner JD, Carruth BR, Wendy B, Ziegler PJ: Children's food preferences: a longitudinal analysis. J Am Diet Assoc 2002;102:16381647.

4 de Lauzon-Guillain B, Jones L, Oliveira A, et al: The influence of early feeding practices on fruit and vegetable intake among preschool children in 4 European birth cohorts. Am J Clin Nutr 2013;98:804-812.

5 Jones L, Moschonis G, Oliveira A, et al: The influence of early feeding practices on healthy diet variety score among pre-school children in four European birth cohorts. Public Health Nutr 2015;18:1774-1784.

6 Gerrish CJ, Mennella JA: Flavor variety enhances food acceptance in formula-fed infants. Am J Clin Nutr 2001;73:1080-1085.

7 Maier AS, Chabanet C, Schaal B, Leathwood $\mathrm{PD}$, Issanchou $\mathrm{SN}$ : Breastfeeding and experience with variety early in weaning increase infants' acceptance of new foods for up to two months. Clin Nutr 2008;27:849-857.

8 Lange C, Visalli M, Jacob S, Chabanet C, Schlich P, Nicklaus S: Maternal feeding practices during the first year and their impact on infants' acceptance of complementary food. Food Qual Pref 2013;29:89-98.

9 Fildes A, Lopes C, Moreira P, et al: An exploratory trial of parental advice for increasing vegetable acceptance in infancy. Br J Nutr 2015;114:328-336.
10 Ahern SM, Caton SJ, Blundell P, Hetherington MM: The root of the problem: increasing root vegetable intake in preschool children by repeated exposure and flavour flavour learning. Appetite 2014;80:154-160.

11 Bouhlal S, Issanchou S, Chabanet C, Nicklaus S: 'Just a pinch of salt'. An experimental comparison of the effect of repeated exposure and flavor-flavor learning with salt or spice on vegetable acceptance in toddlers. Appetite 2014;83:209-217.

12 Caton SJ, Ahern SM, Remy E, Nicklaus S, Blundell $\mathrm{P}$, Hetherington MM: Repetition counts: repeated exposure increases intake of a novel vegetable in UK pre-school children compared to flavour-flavour and flavour-nutrient learning. Br J Nutr 2013;109:20892097.

13 de Wild V, de Graaf C, Jager G: Efficacy of repeated exposure and flavour-flavour learning as mechanisms to increase preschooler's vegetable intake and acceptance. Pediatr Obes 2015;10:205-215.

14 de Wild VW, de Graaf C, Jager G: Effectiveness of flavour nutrient learning and mere exposure as mechanisms to increase toddler's intake and preference for green vegetables. Appetite 2013;64:89-96.

15 Hausner H, Olsen A, Møller P: Mere exposure and flavour-flavour learning increase 2-3 year-old children's acceptance of a novel vegetable. Appetite 2012;58:1152-1159.

16 Remy E, Issanchou S, Chabanet C, Nicklaus S: Repeated exposure of infants at complementary feeding to a vegetable puree increases acceptance as effectively as flavor-flavor learning and more effectively than flavor-nutrient learning. J Nutr 2013;143:1194-1200.
17 Caton SJ, Blundell P, Ahern SM, et al: Learning to eat vegetables in early life: the role of timing, age and individual eating traits. PloS one 2014;9:e97609.

18 Birch LL: Effects of peer models' food choices and eating behaviors on preschoolers' food preferences. Child Dev 1980;51:489496.

19 Zeinstra GG, Kooijman V, Kremer S: My idol eats carrots, so do I? The delayed effect of a classroom-based intervention on 4-6-yearold children's intake of a familiar vegetable. Food Qual Pref 2016, http://dx.doi.org/ 10.1016/j.foodqual.2016.11.007.

20 Rohlfs Domínguez P, Gámiz F, Gil M, et al: Providing choice increases children's vegetable intake. Food Qual Pref 2013;30:108113

21 Zeinstra GG, Renes RJ, Koelen MA, Kok FJ, de Graaf C: Offering choice and its effect on Dutch children's liking and consumption of vegetables: a randomized controlled trial. Am J Clin Nutr 2010;91:349-356.

22 de Wild VW, de Graaf C, Boshuizen HC, Jager G: Influence of choice on vegetable intake in children: an in-home study. Appetite 2015; 91:1-6.

23 Remy E, Issanchou S, Chabanet C, Boggio V, Nicklaus S: Impact of adiposity, age, sex and maternal feeding practices on eating in the absence of hunger and caloric compensation in preschool children. Int J Obes (Lond) 2015; 39:925-930.

24 Collective Work of the Consortium Habeat (FP7-245012-HabEat): Vegetables and Fruits - Help Your Child to Like Them - A Guide for Parents of Young Children. St Luce sur Loire, 2014. 